\title{
Die Notwendigkeit der psychosozialen Begleitung von Eltern in der Neonatologie
}

\author{
The Need for Psychosocial Support of Parents of Children in \\ Neonatal Care
}

\author{
Autoren \\ Nicole Schäfer, Harald Karutz, Olaf Schenk
}

Institute

Department Pädagogik, Pflege und Gesundheit, MSH

Medical School Hamburg

\section{Schlüsselwörter}

Psychoneonatologie, psychosoziale Begleitung, Risiko- und Schutzfaktoren, Eltern-Kind-Beziehung

Key words

neonatal psychology, psychosocial support, risk and protective factors, parent-child-relationship

eingereicht 21.11.2016

angenommen nach Überarbeitung 19.04.2017

Bibliografie

DOI https://doi.org/10.1055/s-0043-110056

Online-Publikation: 7.6.2017

Z Geburtsh Neonatol 2017; 221: 217-225

(c) Georg Thieme Verlag KG Stuttgart · New York ISSN 0948-2393

Korrespondenzadresse

Nicole Schäfer

Department Pädagogik, Pflege und Gesundheit

MSH Medical School Hamburg

Am Kaiserkai 1

20457 Hamburg

info@medicalschool-hamburg.de

\section{ZUSAMMENFASSUNG}

Hintergrund Erhebliche Fortschritte in der neonatologischen Medizin haben in den letzten Jahren zu einer Verringerung der Mortalitätsrate und zu einem Anstieg der Morbiditätsrate unter den zu früh- und risikogeborenen Kindern geführt. Häufig ist diese Entwicklung jedoch mit einer zunehmenden Belastung für die gesamte Familie, insbesondere für die Eltern, verbunden. Anhand einer umfangreichen Literaturarbeit wird daher die Notwendigkeit der psychosozialen Begleitung von Eltern in der Neonatologie herausgestellt.

Methodik In den Datenbanken Pubmed, Psyndex, CINAHI und medpilot wurde zunächst eine systematische Literaturrecher- che durchgeführt. Um weitere relevante Publikationen zu finden, wurden außerdem die Referenzlisten der gefundenen Artikel überprüft. Eine anschließende Freitextsuche hatte die Ergänzung der Schlagwortsuche zum Ziel. Insgesamt konnten auf diese Weise 78 Veröffentlichungen aus den Jahren von 1975 bis 2015 in die Studie einbezogen werden.

Ergebnisse Die Bedeutung der psychosozialen Begleitung von Eltern in der Neonatologie lässt sich anhand der ausgewerteten Fachliteratur eindrucksvoll belegen. Allerdings fällt auf, dass die Notwendigkeit eines Assessments als Grundlage einer den individuellen Bedürfnissen der Familie entsprechenden Begleitung zwar bekannt ist, die Verwendung von validierten Screeninginstrumenten offenbar jedoch noch nicht zum Standard gehört. Ferner wird deutlich, dass der Bedarf an psychosozialer Unterstützung seitens der Eltern nicht allein vom medizinischen Risiko des Kindes abhängt. Schlussfolgerungen Die vorliegenden Erkenntnisse implizieren insbesondere die Herausforderung, unter Berücksichtigung der in der Entwicklung befindlichen Eltern-Kind-Einheit, in einem Gesundheitssystem mit eingeschränkten Ressourcen und vor dem Hintergrund gesundheitsökonomischer Vorgaben, dennoch eine hoch individuelle Betreuung betroffener Familien möglich zu machen. Mitunter intuitiv ausgebildete Konzepte sollten in Zukunft weiter wissenschaftlich fundiert werden. Beiträge aus den Bereichen der Psychologie und Bindungsforschung sind dabei zu berücksichtigen.

\section{ABSTRACT}

Background Advances in neonatal care have reduced mortality but increased morbidity in babies born pre-maturely or after highrisk pregnancies. However, this often increases the burden on the family and the parents in particular. A systematic review of the literature was conducted that demonstrated the importance of psychosocial support for parents of children in neonatal care.

Methods A systematic search of Pubmed, Psyndex, CINAHI and medpilot was conducted. Reference lists of the included articles were also searched for relevant publications. A free-text search found further publications. Together, 78 publications (from 1975-2015) were included in our review.

Results A shift from a biomedical model and child-centred treatment to family-centred care has already taken place in neonatal care. However, there is still a considerable gap bet- 
ween theory and practice. Although there is awareness of the need for psychosocial support of parents, the focus of day-to-day care is still on medical interventions and life-supporting treatment for the child. In particular, while the importance of an assessment of needs as a basis for family-centred psychosocial support appears to be wellknown, validated screening instruments are rarely used. In addition, the demand for psychosocial support of parents is not just solely determined by the child's medical risk.
Conclusions The results highlight the challenges of delivering individualised psychosocial support to families within a healthcare system of limited resources, with practitioners having to take into account the developing parent-child relationship as well as health economics. In future, psychosocial support should be based on evidence rather than intuition. Attachment theory and research, and health psychology can contribute to this development.

\section{Einleitung}

Erhebliche Fortschritte in der medizinisch-technischen Versorgung haben in den vergangenen Jahren dazu geführt, dass immer mehr früh- und risikogeborene Kinder überleben [1-4]. Oft geht diese positive Entwicklung jedoch mit einer erhöhten psychosozialen Belastung für die betroffenen Familien einher [5, 6]. Der Aufbau einer stabilen Eltern-Kind-Bindung ist aufgrund der besonderen Umstände häufig erschwert [7], sodass neben der neonatologischen Versorgung psychosoziale Unterstützungsangebote wünschenswert sind.

In einigen Kliniken werden solche Unterstützungsangebote als Reaktion auf das zunehmende Bewusstsein von elterlichen Belastungen auch bereits umgesetzt [2-4, 8, 9, 20, 45, 62, 63]. In der Regel sind sie jedoch sehr allgemein umschrieben und es erfolgt keine Darstellung spezifischer Maßnahmen oder konkreter Wirkungsweisen [10]. „Stattdessen ist weitgehend unbekannt, wie viele Kliniken in welchem Ausmaß Stellen zur psychosozialen Versorgung eingerichtet haben oder dies planen, wie diese finanziert werden und mit welchem Umfang, nach welchen Grundsätzen, mit welchem Berufsabschluss und Ausbildungshintergrund die Stelleninhaber arbeiten“ [11]. Angesichts wirtschaftlicher Aspekte werden die bereits implementierten Unterstützungsangebote zudem meist ausschließlich den durch das neonatologische Behandlungsteam subjektiv als stark belastet eingeschätzten Eltern zur Verfügung gestellt. Dieses Hilfsangebot entspricht in vielen Fällen jedoch nicht dem tatsächlichen Bedarf der betroffenen Familien [9].

\section{Zielsetzung und Methodik}

Basierend auf den Befunden aus Bindungsforschung und Entwicklungspsychologie geht es in diesem Review um die Notwendigkeit der psychosozialen Begleitung von Eltern in der Neonatologie. Die Erhebung des Datenmaterials erfolgte auf Basis einer systemati- schen, recht komplexen Literaturrecherche zu einer Vielzahl von Suchbegriffen wie z. B. Neonatologie (neonatology), Eltern (parents) bzw. Vater (father), Mutter (mother) und Familie (family); psychosoziale Intervention (psychosocial intervention), Familienunterstützung (family support intervention) sowie der Verbindung dieser Wörter mithilfe der Boolschen Operatoren in den Datenbanken Pubmed und Psyndex, ebenso wie CINAHI und medpilot. Google Scholar wurde allein für eine orientierende Suche genutzt.

Auch die Referenzlisten der bereits gefundenen Artikel wurden überprüft, um auf diese Weise weitere Artikel zu finden, die die Suchkriterien (Inhaltliche Relevanz, Sprachen Englisch und Deutsch, Erscheinungsjahre 1975-2015) erfüllen. Eine anschließende Freitextsuche hatte die Ergänzung der Schlagwortsuche zum Ziel. Insgesamt wurden auf diese Weise letztlich 78 Publikationen ausgewertet. Der vorliegende Beitrag stellt ausgewählte Erkenntnisse aus der Untersuchung in einem zusammenfassenden Überblick dar.

\section{Entwicklungspsychopathologische Grund- lagen}

Die Gebiete der Entwicklungspsychologie und klinischen Psychologie wurden in den letzten Jahrzehnten vorrangig als 2 nicht miteinander vereinbare Disziplinen angesehen. Dabei verbindet die Entwicklungspsychopathologie diese beiden Disziplinen. Sie beruht auf einer biopsychosozialen Sichtweise und nutzt diese, um etwaige Risiken für die kindliche Entwicklung zu identifizieren . In den Fokus der Bindungsforschung gelangen daher frühkindliche Beziehungsund Bindungserfahrungen sowie ihr Einfluss auf die weitere Entwicklung des Kindes. Um ergründen zu können, warum diese ungünstigen Bedingungen entstehen, müssen frühe Ausgangssituationen in Entwicklungsprozessen genauer betrachtet werden [12].

- Tab. 1 Risikofaktoren in der frühkindlichen Entwicklung [13].

Biologisch-medizinische Faktoren

- Pränatale Faktoren (Vorerkrankung der Mutter, andauernde psychische Belastungen, Substanzkonsum)

- Perinatale Faktoren (Geburtskomplikationen wie vorzeitige Wehen, vorzeitiger Blasensprung, Geburtsstillstand)

- Postnatale Faktoren (Frühgeburt, Erkrankung des Neugeborenen, intensivmedizinische Behandlung)

\section{Faktoren innerhalb der Eltern-Kind-Interaktion}

- Bindungsverhalten (unsichere Bindungsformen), fehlende Feinfühligkeit der Bindungsperson, psychische Störungen der Eltern

Familiäre und sozio-demografische Faktoren

- Konflikte und Vorerfahrungen der Eltern, innerfamiliäre Gewalt, frühe oder späte Elternschaft, niedriger sozioökonomischer Status 
- Tab. 2 Schutzfaktoren in der frühkindlichen Entwicklung [13].

\section{Umgebungsbezogene Schutzfaktoren}

- Schutzfaktoren innerhalb der Familie (Stabile emotionale Beziehung zu einer Bezugsperson, familiärer Zusammenhalt, Modelle positiven Bewältigungsverhaltens, hoher sozioökonomischer Status)

- Schutzfaktoren innerhalb des sozialen Umfeldes (adäquate Erstversorgung und Pflege, soziale Unterstützung)

- Tab. 3 Phasenmodell nach Gloger-Tippelt [19].

bis 12. SSW Verunsicherungsphase

12.-20. SSW Anpassungsphase

20.-32. SSW Konkretisierungsphase

32. SSW bis Antizipations- und

zur Geburt Vorbereitungsphase
Starke körperliche Veränderungen, kognitive Auseinandersetzung, emotionale Ambivalenzen Kognitive Akzeptanz der Schwangerschaft, umfassende Reflexion, Reduktion von Ambivalenzen

Konkretisierung der Schwangerschaft, direkte Auseinandersetzung mit dem Kind, Grundlage für Bindungsaufbau nach Geburt

Psychische Vorbereitung auf bevorstehende Geburt und Übernahme der Elternrolle, körperliches Unwohlsein, emotionale Ambivalenzen

\section{Risiko- und Schutzfaktoren in der frühkindlichen Entwicklung}

Mit Bezug auf eine biopsychosoziale Sichtweise gerät v. a. das Risiko- und Schutzfaktorenkonzept in den Fokus der Betrachtung von Entwicklungsprozessen. Insbesondere die erste Zeit im Leben stellt für das sich entwickelnde Kind einen äußerst vulnerablen Lebensabschnitt dar, in dem es zahlreichen Risikofaktoren ausgesetzt ist. Für zu früh und kranke neugeborene Kinder lassen sich biologischmedizinische Risiken, Risiken innerhalb der Eltern-Kind-Interaktion und familiäre sowie sozio-demografische Risiken unterscheiden [13] ( Tab. 1).

Neben den Faktoren, die das Risiko für eine Abweichung von der gesunden Entwicklung erhöhen, gibt es überdies Bedingungen, die eine eher risikomindernde und schützende bzw. protektive Wirkung haben. Dabei lassen sich die für zu früh- und risikogeborene Kinder bedeutende Schutzfaktoren in protektive Faktoren innerhalb der Familie und protektive Faktoren innerhalb des sozialen Umfeldes differenzieren [13] ( Tab. 2).

Entscheidend ist, dass einige Risikobedingungen nur zu bestimmten Zeitpunkten Einfluss auf die kindliche Entwicklung haben, andere Faktoren hingegen können sich über den gesamten Entwicklungsverlauf auswirken [13].

\section{Resilienz zur Unterstützung der kindlichen Entwicklung}

Im direkten Zusammenhang mit dem Risiko- und Schutzfaktorenkonzept steht das Konzept der Resilienz [13]. Im Gegensatz zur Vulnerabilität (Verletzlichkeit) steht Resilienz für die Widerstandsfähigkeit gegenüber ungünstigen Entwicklungs- und Belastungsfaktoren [14-16]. Die Fähigkeit zur Resilienz lässt sich auf dynamische und transaktionale Anpassungs- und Entwicklungsprozesse zurückführen und zeigt somit, dass neben den individuellen Ressourcen gerade im Bereich der Neonatologie die sozialen, umgebungsbezogenen Schutzfaktoren, wie eine stabile emotionale Beziehung zu einer Bezugsperson, von besonderer Bedeutung sind [13, 17].

\section{Eltern zu früh- und risikogeborener Kinder}

Um eine langfristig bestmögliche Entwicklung für die Kinder realisieren zu können, sind in der Neonatologie daher nicht nur kindzentrierte Interventionen relevant. Gleichermaßen nehmen elternzentrierte Interventionen einen hohen Stellenwert ein, denn auch die Eltern benötigen eine individuelle Begleitung [18]. Ziel dieser Begleitung sollte die Entwicklung passgenauer Angebote sein, um auch die Eltern als Empfänger von Versorgungsleistungen in der Neonatologie mit einzubeziehen [19].

Gemäß dem Phasenmodell von Gloger-Tippelt ( $>$ Tab. 3) vollziehen sich während des gesamten Schwangerschaftsprozesses Veränderungen auf physischer, psychischer und sozialer Ebene, die sowohl einer kognitiven und emotionalen als auch einer aktiven Bewältigung und Adaption bedürfen [20]. Diese parallel zur kindlichen Entwicklung verlaufenden psychischen Prozesse der werdenden Eltern dienen dabei der Vorbereitung auf die bevorstehende Geburt und die zukünftige Elternschaft. Der idealtypische psychische Prozess bildet diesbezüglich die Grundlage für einen feinfühligen Umgang der Eltern mit ihrem Kind [19, 20]. Eltern zu früh geborener Kinder können diesen entscheidenden Adaptionsprozess durch die zu frühe Geburt jedoch nicht vollständig im Vorfeld durchlaufen. Ihnen fehlt wertvolle Zeit der Vorbereitung [21, 22]. Zumindest ein Teil der Adaption an die Elternschaft kann - unter meist bedeutend schwierigeren Umständen - erst auf der neonatologischen Intensivstation erfolgen [23].

\section{Früh- oder Risikogeburt als kritisches Lebensereignis der Eltern}

In den letzten Jahren konnte belegt werden, dass die zu frühe oder komplikationsbehaftete Geburt ein kritisches Lebensereignis für Eltern darstellt [24, 25]. Auch der Krankenhausaufenthalt eines Kindes auf der Neugeborenenintensivstation ist für die Eltern ein potenziell traumatisches Ereignis, in dessen Folge eine akute Belastungsreaktion auftreten kann [26]. Ausgelöst wird diese durch das subjektive Gefühl von Lebensbedrohung, Ohnmacht, Hilflosigkeit und Kontrollverlust [27]. 
- Tab. 4 Risiko- und Schutzfaktoren der Eltern [14, 31, 32].

\begin{tabular}{|c|c|c|}
\hline Tendenziell risikoverstärkende Wirkung & Variable Faktoren & Tendenziell schützende Wirkung \\
\hline \multicolumn{3}{|l|}{ Vorbedingungen } \\
\hline $\begin{array}{l}\text { Zu hohes oder zu niedriges Alter, niedriger } \\
\text { sozioökonomischer Status }\end{array}$ & $\begin{array}{l}\text { Soziodemografische } \\
\text { Variablen }\end{array}$ & $\begin{array}{l}\text { Mittleres Alter, mittlerer bis hoher sozioökonomi- } \\
\text { scher Status }\end{array}$ \\
\hline Kritisch, unbewältigt & $\begin{array}{l}\text { Vorangegangenev } \\
\text { Lebensereignisse }\end{array}$ & Unkritisch, bewältigt \\
\hline Emotionale Labilität, Vulnerabilität & Persönlichkeitsfaktoren & $\begin{array}{l}\text { Emotionale Stabilität } \\
\text { Resilienz }\end{array}$ \\
\hline $\begin{array}{l}\text { Komplikationsbehaftet, Vorbereitung auf Elternschaft } \\
\text { unterbrochen }\end{array}$ & Schwangerschaftsverlauf & $\begin{array}{l}\text { Komplikationsfrei, Vorbereitung auf Elternschaft } \\
\text { abgeschlossen }\end{array}$ \\
\hline $\begin{array}{l}\text { Einsatz von } \\
\text { Reproduktionsmedizin, vorangegangene Frühgeburt, } \\
\text { vorhergehender Schwangerschaftsabbruch }\end{array}$ & Spezifische Faktoren & Natürliche Schwangerschaft, natürliche Geburt \\
\hline \multicolumn{3}{|l|}{ Situative Faktoren } \\
\hline Früh- oder Risikogeburt & Ablauf der Geburt & Komplikationslose Geburt \\
\hline Kritisch & Gesundheitszustand der Mutter & unkritisch \\
\hline Zu früh oder krankes, neugeborenes Kind & Gesundheitszustand des Kindes & Gesundes, zum Termin geborenes Kind \\
\hline Vorhanden & Dissoziative Zustände & Nicht vorhanden \\
\hline $\begin{array}{l}\text { Negativ, hohe Stressbelastung, wahrgenommene } \\
\text { Bedrohung des Kindes, }\end{array}$ & $\begin{array}{l}\text { Subjektive Interpretation und } \\
\text { Bewertung }\end{array}$ & $\begin{array}{l}\text { Positiv, niedrige Stressbelastung } \\
\text { Keine } \\
\text { wahrgenommene } \\
\text { Bedrohung des Kindes }\end{array}$ \\
\hline \multicolumn{3}{|l|}{ Stationäre Einflussfaktoren } \\
\hline $\begin{array}{l}\text { Selbstbeschuldigungen und Schamerleben, keine } \\
\text { wahrgenommene Kontrolle, keine soziale Unterstützung }\end{array}$ & $\begin{array}{l}\text { Elterliche } \\
\text { Bewältigungsstrategien }\end{array}$ & $\begin{array}{l}\text { Informationssuche } \\
\text { und Erfahrungsaustausch, wahrgenommene } \\
\text { Kontrolle, soziale Unterstützung }\end{array}$ \\
\hline $\begin{array}{l}\text { Zusätzliche } \\
\text { Belastungsfaktoren }\end{array}$ & Familienleben & $\begin{array}{l}\text { Keine zusätzlichen } \\
\text { Belastungsfaktoren }\end{array}$ \\
\hline $\begin{array}{l}\text { Standardversorgung } \\
\text { Medizintechnik, Trennung vom Kind, Unsicherheit }\end{array}$ & $\begin{array}{l}\text { Neonatologische } \\
\text { Intensivstation }\end{array}$ & $\begin{array}{l}\text { Entwicklungs- und beziehungsorientierte Pflege, } \\
\text { psychosoziale Begleitung }\end{array}$ \\
\hline
\end{tabular}

Eltern, deren Kind auf einer neonatologischen Intensivstation behandelt wurde, haben zudem ein höheres Risiko, an einer Depression oder einer posttraumatischen Belastungsstörung zu erkranken als Eltern von gesunden Kindern [26, 28]. Der mit dem Krankenhausaufenthalt des Kindes verbundene Stress kann zu einer Beeinflussung des elterlichen Verhaltens sowie zu einer Erzeugung langfristiger emotionaler Probleme und zu gesundheitlichen Veränderungen führen [29]. Ist es den Eltern nicht möglich, die traumatischen Erfahrungen rund um die Geburt zu verarbeiten, sind sie für das Kind z. B. emotional nicht verfügbar [30]. Zudem können andere, bereits (selbst)erfahrene Trennungs- und Verlustereignisse durch die zu frühe oder komplikationsbehaftete Geburt reaktiviert werden. Bekommen die Eltern in der Auseinandersetzung mit dieser vergangenen Problematik keine professionelle Unterstützung, ist es möglich, dass der Aufbau einer tragfähigen Eltern-Kind-Bindung erschwert wird [31].

\section{Resilienzforschung im Erwachsenenalter}

Vor dem Hintergrund der bisherigen Darstellung wird deutlich, dass eine zu frühe oder komplikationsbehaftete Geburt nicht nur für das Kind, sondern auch für die Eltern mit der Aktivierung von Schutzund Risikofaktoren einhergeht [31]. Aktuelle Forschungsergebnis- se stellen die erfolgreiche Bewältigung stressreicher und potenziell traumatischer Ereignisse als entscheidend heraus [14].

Weniger als auf die Intensität einzelner Stressoren kommt es allerdings auf ihre subjektive Wahrnehmung, Interpretation und Bewertung an. Aus diesem Grund führt allein das Vorhandensein eines Schutzfaktors auch nicht automatisch zu einer Immunität oder Resistenz gegenüber Stressoren. Ein oder mehrere Schutzfaktoren bewirken eher eine Reduktion der Wahrscheinlichkeit für ein nicht förderliches Bewältigungsverhalten [14].

Insbesondere für die Arbeit mit Eltern in der Neonatologie ist dieses Wissen äußerst bedeutsam, denn die Situation mit einem zu früh geborenen oder kranken Kind bringt eine Vielzahl medizinischer und psychologischer Risiken mit sich [32]. Folglich verstärkt sie die Verletzlichkeit der ganzen Familie.

\section{Risiko- und Schutzfaktoren der Eltern}

Nicht alle Eltern haben einen intensiven Unterstützungsbedarf [21]. Vor dem Hintergrund der aktuellen Erkenntnisse zur Vielfalt der unterschiedlichen Belastungsfolgen einer zu frühen und komplikationsbehafteten Geburt scheint es jedoch sinnvoll zu sein, die unterschiedlichen Einflussfaktoren zu identifizieren, die in Form von risikoerhöhenden und schützenden Variablen die Bewältigung dieser erschweren oder erleichtern können [33] (• Tab. 4). 


\section{Die Bedeutung der Eltern-Kind-Beziehung}

Zentrale Erkenntnisse der Bindungstheorie und -forschung zeigen, dass die Qualität der Eltern-Kind-Beziehung eine gewisse Schlüsselfunktion in der Entwicklung eines Kindes übernimmt. Eine positive Eltern-Kind-Beziehung dient der Entwicklungsförderung und kann aus diesem Grund als entscheidende Ressource in der Entwicklung eines Kindes gesehen werden [34]. V. a. im frühen Kindesalter liegen die auf die Entwicklung einflussnehmende Faktoren oft nicht ausschließlich bei dem Kind, sondern sind darüber hinaus in der Qualität der einander beeinflussenden Beziehungen zwischen dem Kind und seinen Bezugspersonen zu finden [12].

Alle Kinder entwickeln eine Bindung zu einer Bezugsperson [35], jedoch kann jede Bindung dabei unterschiedliche Qualität annehmen [37]. Das medizinische Risiko durch die Unreife oder Erkrankung des Kindes kann diesbezüglich Auswirkungen auf die Qualität der Mutter-Kind-Interaktion und Bindungsrepräsentation der Bezugsperson haben [1]. Die für die Ausbildung des kindlichen Bindungsstils bedeutenden Verhaltensweisen der Bezugsperson werden dabei unter dem Aspekt der Feinfühligkeit zusammengefasst [38].

Eine feinfühlige Bezugsperson kann die Signale ihres Kindes wahrnehmen, weiß diese sowohl zutreffend zu interpretieren als auch angemessen, zuverlässig und prompt darauf zu reagieren [38]. So kann vermutet werden, dass ein feinfühliges Verhalten der Bezugsperson Einschränkungen in der Interaktion, bedingt durch die Unreife oder Erkrankung ihres Kindes, kompensiert [5]. Es ist jedoch davon auszugehen, dass das Fürsorgesystem bei einer zu frühen Geburt noch nicht vollständig entwickelt und aus diesem Grund nicht abrufbar ist. Zudem kann das Kind angesichts seiner psychophysiologischen Unreife nur begrenzt eindeutig interpretierbare Signale aussenden, gleichwohl diese den Zugang der Mütter zu ihrem Fürsorgesystem unterstützen würden [2]. Darüber hinaus hat ein langer Krankenhausaufenthalt zur Folge, dass sich beide bindungsrelevanten Systeme - sowohl das mütterliche Fürsorgesystem als auch das kindliche Bindungssystem - nur unter äußerst schwierigen Bedingungen entwickeln können [39].

Eine Fehlinterpretation der gesandten kindlichen Signale und Bedürfnisse kann wiederum zu einem negativen, wechselseitigen Interaktionskreislauf führen und dadurch den Ausbau einer nicht förderlichen Beziehungsdynamik unterstützen [36]. Die Bindungsorganisation hat demnach einen entscheidenden Einfluss auf die weitere Entwicklung des Kindes und stellt einen möglichen Schutzbzw. Risikofaktor dar [40].

Entscheidend für den Aufbau einer positiven Eltern-Kind-Interaktion ist v. a. die erste Zeit nach der Geburt. Gerade in dieser finden für die Entwicklung der Bindung zwischen Eltern und Kind wichtige physiologische Prozesse statt. Die mit der intensivmedizinischen Behandlung verbundene Trennung von Eltern und Kind findet somit in einer sehr sensiblen Phase des Bindungsaufbaus statt [41]. Zudem kann die durch die zu frühe Geburt bedingte sensorische Unreife des Kindes das Aussenden von eindeutig zu interpretierenden Signalen und das angemessene Reagieren auf elterliche Signale beträchtlich erschweren [42]. Der vorzeitige Abbruch des Eltern-Werdungs-Prozesses in der Schwangerschaft und die postnatale Notwendigkeit eines intensivmedizinischen Aufenthaltes des Kindes können sich zeitgleich nachhaltig negativ auf den Auf- und Ausbau der ElternKind-Interaktion auswirken $[41,43]$.

\section{Das transaktionale Modell}

Die Verdeutlichung der zuvor beschriebenen Wechselwirkungen zwischen den einzelnen Faktoren gelingt besonders gut in transaktionalen Modell von Suess und Zimmermann [16]. In diesem Modell wird zunächst zwischen 3 unterschiedlichen Systemebenen unterschieden: Die Ebene des Kindes, die der Eltern und die der Umwelt ( $\triangleright$ Abb. 1).

Das aus den Modellen resultierende Verständnis über einzelne Entwicklungsverläufe, Interaktionsprozesse und Wirkungsweisen von Schutz- und Risikofaktoren ist außerordentlich wichtig, weil sich ausschließlich darüber notwendige Interventionen zielgerichtet planen und steuern lassen [16].

\section{Psychosoziale Begleitung in der Neonato- logie}

In der aktuellen Forschungslage zur psychosozialen Begleitung von Eltern in der Neonatologie zeigt sich eine eher widersprüchliche Datenlage. Einerseits besteht Konsens über die Notwendigkeit der psychosozialen Begleitung und die besondere Bedeutung der Eltern für ihr zu früh oder krank geborenes Kind als protektiver Faktor $[13,40]$. Andererseits wird bei genauer Betrachtung der Studienlage deutlich, dass es keine einheitliche Begriffsklärung gibt und demzufolge keine einheitliche Definition vorliegt [44]. Häufig existieren lediglich Aufzählungen von einzelnen Maßnahmen, die eine psychosoziale Begleitung umfassen soll. So bleibt der Begriff der psychosozialen Begleitung inhaltlich ungenau.

Aufgrund der fehlenden Definition und dem damit nicht einheitlichen Verständnis von psychosozialer Begleitung ist es ein notwendiger Schritt, eine übergeordnete Referenz zu finden. Vor dem Hintergrund des in der Einleitung beschriebenen Paradigmenwechsels und der damit verbundenen, zunehmend systemorientierten Sichtweise der Experten scheint die Definition von Nestmann diesbezüglich geeignet zu sein. Sie umfasst alle notwendigen Begrifflichkeiten und schließt darüber hinaus den biopsychosozialen Ansatz mit ein. Auf diese Weise wird neben dem Kind auch dessen Familie in gleichem Maße berücksichtigt:

„Eine professionelle Unterstützungsleistung, die in einem gemeinsamen Prozess der Orientierung, Planung, Entscheidung und Handlung versucht, biopsychosoziale Ressourcen von Personen [...] zu entdecken, zu fördern, zu erhalten und aufeinander zu beziehen. Ziel psychosozialer Beratung ist es Entfaltung von Einzelnen in formellen und informellen sozialen Systemen zu ermöglichen, selbstbestimmtes und selbstkontrolliertes Gestalten von Alltag und Leben, die Bearbeitung ihrer Anforderungen und die Nutzung ihrer Entwicklungschancen zu sichern sowie Belastungen und Krisen zu verhindern, möglichst früh anzugehen, sowie deren Folgen für Personen und Systeme konstruktiv zu bewältigen “ [45].

Um die Bewältigungs- und Adaptionsprozesse der Eltern unterstützen zu können und die Auswirkungen dieses kritischen Lebensereignisses zu reduzieren, ist demnach eine psychosoziale Begleitung notwendig $[21,46]$. Ziele dieser sind $u$. a. die verbesserte Beziehungsaufnahme der Eltern zu ihrem Kind und die Förderung des 


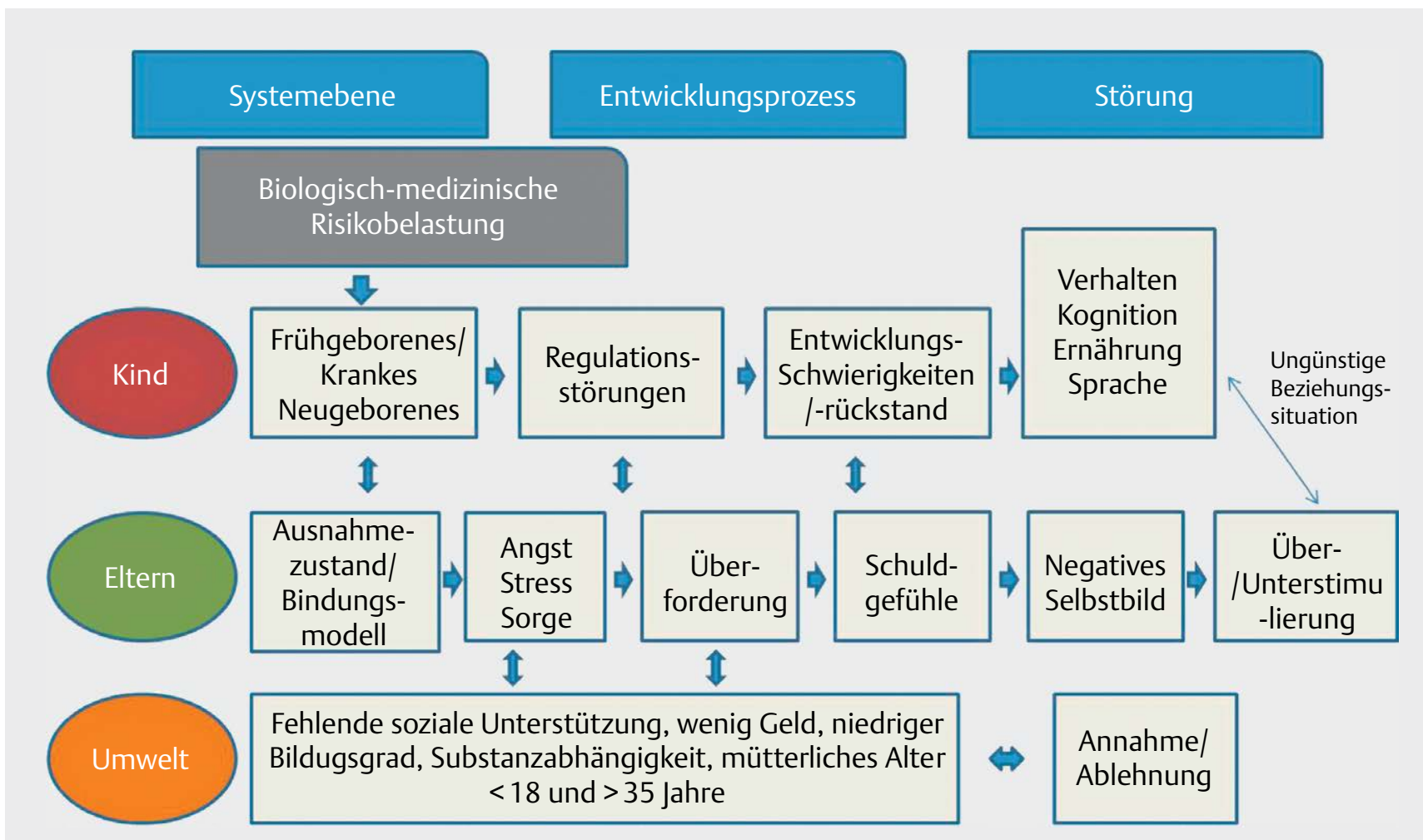

Abb. 1 Transaktionales Modell [ modifiziert nach 16].

Aufbaus einer tragfähigen Bindung als bestmögliche Grundlage für die Entwicklung ihres Kindes [18, 21, 30,47]. Um ein möglichst effizientes Betreuungskonzept anbieten zu können, ist eine psychosoziale Begleitung als integrativer Bestandteil der neonatologischen Versorgung sinnvoll [46].

\section{Einschätzung des elterlichen Bedarfs an professionel- ler Unterstützung}

Für die fachliche Kommunikation und Reflexion sowie für die Konzeptualisierung elternorientierter Unterstützungsangebote sind wissenschaftlich fundierte Kenntnisse über den Unterstützungsbedarf der Eltern notwendig. Aus diesem Grund stellt sich die Frage, nach welchen Kriterien die professionelle Einschätzung des elterlichen Bedarfs erfolgt [48]. Bezüglich der Einschätzung von individuellen und familiären Bedürfnissen dient ein Assessment als Basis für die Entwicklung und Implementierung geeigneter Maßnahmen in Verbindung mit beratungsrelevanten Aspekten aus der Medizin und Pflege [49].

Im Leitfaden des Frühgeborenenverbandes zur Elternberatung in der Neonatologie [44] erklären die Autoren, dass für die Erstellung eines auf die Eltern zugeschnittenen Bedarfsplanes zurzeit ausschließlich allgemein gültige Assessmentinstrumente vorliegen, die die Bedürfnisse der Eltern auf einer neonatologischen Intensivstation nicht in vollem Ausmaß widerspiegeln. In dem Artikel von Hynan et al. [26] hingegen stellen die Autoren eine Reihe validierter Screeninginstrumente zur Einschätzung von Stress und posttraumatischer Belastung in Verbindung mit der Erforschung von beeinflussenden Risikofaktoren dar. Diese sind insbesondere für den Einsatz bei Eltern mit hochrisikogefährdeten Kindern geeignet. Insgesamt empfehlen die Autoren Screeningverfahren stärker zu berücksichtigen, da diese helfen können elterliche traumati- sche Reaktionen zu erfassen und auf diese Weise negative Auswirkungen auf die kindliche Entwicklung durch früh einsetzende präventive Maßnahmen zu minimieren [26]. Andere Autoren nutzen weitere Assessmentinstrumente, die bereits in vorhergegangenen Untersuchungen den größten klinischen Nutzen gebracht haben [50]. So können an dieser Stelle beispielhaft die Parental Stressor Scale [51] und das Clinical Interview for Parents of High-Risk Infants [52] genannt werden.

In deutschsprachigen Studien wurde der elterliche Unterstützungsbedarf während des stationären Aufenthaltes ihres Kindes bisher nurvereinzelt und in unsystematischer Form beschrieben [48]. Dennoch wird in den meisten Studien auf die elterliche Belastung hingewiesen [5,42]. Die durch den Übergang zur Elternschaft bedingte elterliche Belastung wird dabei häufig mit entwicklungspsychologischen Theorien, die situative elterliche Belastung eher mit Stress- bzw. Trauma-theoretischen Ansätzen erklärt [48]. In der aus Österreich stammenden Studie zu Belastungen von Eltern auf neonatologischen Intensivstationen erfolgte die Einschätzung der elterlichen emotionalen Belastungen durch das neonatologische Behandlungsteam. Anhand der gewonnenen Forschungsergebnisse konnten die Autoren zeigen, dass das Ausmaß der psychischen elterlichen Belastungen unabhängig von der medizinischen Gefährdung des Kindes war. Darüber hinaus gab es nur eine geringe Übereinstimmung zwischen den elterlichen Selbstbeurteilungen und der Fremdbeurteilung durch das neonatologische Betreuungsteam [9]. Dies bestätigen auch Mok \& Leung [53]: „The conclusion drawn from the study is that parents desired more nursing support than they received."

Entscheidend ist, dass sich die Bedürfnisse der Eltern während der Begleitung verändern können. Um genau diese Veränderungen berücksichtigen, elterliche Bedürfnisse individuell einschätzen und 
mögliche Interventionen anpassen zu können, ist eine regelmäßige Assimilation der Bedarfseinschätzung notwendig [44].

\section{Diskussion}

Das übergeordnete Ziel dieses Beitrags war es, die Notwendigkeit der psychosozialen Begleitung von Eltern in der Neonatologie theoretischanalytisch zu explorieren. Vor diesem Hintergrund konnte eine sichere Eltern-Kind-Bindung als wesentlicher Schutzfaktor für die kindliche Entwicklung identifiziert und damit die besondere Bedeutung der Eltern für die kindliche Entwicklung aufgezeigt werden [13,40]. Zudem gibt es inzwischen Reviews und Metaanalysen, die die besondere Bedeutung der Eltern als Schutzfaktor für ihre zu früh oder krank geborenen Kinder betonen [54, 55]. Vor dem Hintergrund der entwicklungspsychologischen Grundlagen ist eine möglichst früh einsetzende, präventive Unterstützung der Eltern demnach unerlässlich, um entstehende Entwicklungsrisiken für das Kind und seine Familie verringern zu können [8]. V. a. im sozialen und emotionalen Entwicklungsbereich hat die ElternKind-Bindung eine schützende Wirkung und kann auf diese Weise ungünstige psychosoziale Risiken abmildern [56-58].

Auffällig ist allerdings, dass die Notwendigkeit eines Assessments als Grundlage für eine geplante und den individuellen Bedürfnissen der Familie entsprechende Begleitung sehr wohl bekannt ist, die Verwendung von validierten Screeninginstrumenten bislang jedoch nicht zum Standard gehört. Empfehlenswert wäre daher die Entwicklung eines standardisierten Befragungsinstrumentes zur quantitativen Bedarfserhebung im deutschsprachigen Raum [48]. Denn zweifellos stellt die Erfüllung von elterlichen Bedürfnissen eine der wichtigsten Interventionen während des stationären Aufenthaltes ihres Kindes dar, weil dies zu einer Stressreduktion und damit einhergehenden Steigerung des Wohlbefindens auf Seiten der Eltern führt.

Deutlich wurde ferner, dass die Bedürfnisse der Eltern nicht allein vom medizinischen Risiko des Kindes abhängen, sondern vielmehr von ihren eigenen biografischen Erfahrungen in schwierigen Herausforderungen, aber auch von der Unterstützung, die sie in ihrem sozialen Umfeld erhalten [59]. Um die elterliche Belastung zu beschreiben, sind daher neben den stresstheoretischen Ansätzen auch Trauma-theoretische Ansätze zur Planung von Interventionen zu berücksichtigen [48-28]. Aus diesem Grund ist es zwingend erforderlich, oft intuitiv ausgerichtete Konzepte wissenschaftlich zu fundieren und Beiträge aus den Bereichen der Psychologie und Bindungsforschung in die Umsetzung und damit in die Aus- und Weiterbildung von neonatologischen Teams zu integrieren [60].
FAZIT FÜR DIE PRAXIS: DAS KONZEPT DER PSYCHONEONATOLOGIE

Die genannten Erkenntnisse implizieren die Herausforderung, eine individuelle Betreuung, unter Berücksichtigung der Besonderheiten der in der Entwicklung befindlichen Eltern-Kind-Einheit, in einem Gesundheitssystem mit eingeschränkten Ressourcen und gesundheitsökonomischen Vorgaben möglich zu machen [41]. Daher ist es in diesem Zusammenhang überaus wichtig geeignete Screeninginstrumente grundsätzlich bei allen Eltern anzuwenden, um den tatsächlichen Unterstützungsbedarf zu ermitteln. Eben weil so viele innere Faktoren mitspielen, die nach außen für das Behandlungsteam auf den ersten Blick nicht sichtbar sind und die Notwendigkeit der Intervention infolgedessen bei vielen Familien übersehen werden könnte. Mit einem einheitlichen Screening wäre es möglich dies zu umgehen.

Dass neben den physischen Aspekten auch das psychische Wohlbefinden zum Gesund-Sein gehört, wird in der Medizin häufig vernachlässigt [61]. Dennoch ist die psychosoziale Begleitung von Eltern in der Neonatologie, vor dem Hintergrund des gesamten Lebenskontextes und der Lebensperspektive des Kindes, Ausdruck ethischer Verpflichtung [6].

Während der Begriff der Psychoonkologie bereits seit Jahrzehnten in der Medizin etabliert ist, so ist der Begriff der Psychoneonatologie allerdings weder geläufig, noch existiert ein solches, klar definiertes Fachgebiet. Stattdessen ist die psychosoziale Versorgung in der Neonatologie längst noch nicht die Regel, obgleich viele Aspekte der psychosozialen Versorgung in der pädiatrischen Onkologie mit jener in der Neonatologie übereinstimmen [11].

Wird aber doch zunehmend deutlich, dass es auch psychologische Kenntnisse braucht, um einen auf die gesamte Familie abgestimmte Begleitung planen zu können, so sollte das Wissen weiterer Fachdisziplinen in die alltägliche neonatologische Praxis mit einbezogen und unter dem Konzept einer Psychoneonatologie zusammengetragen werden [11].

Interessenkonflikt

Die Autoren geben an, dass kein Interessenkonflikt besteht.

[1] Forcada-Guex M, Borghini A, Pierrehumbert B et al. Prematurity, maternal posttraumatic stress and consequences on the mother-infant relationship. Early Human Development 2010; 87: 21-26

[2] Dinger ]. Betreuung von Frühgeborenen an der Grenze zur Lebensfähigkeit. In: Reichert J, Rüdiger M, (Hrsg.). Psychologie in der Neonatologie. Psychologisch-sozialmedizinische Versorgung von Familien Frühgeborener. Göttingen: Hogrefe; 2013: 11-28

[3] Schott C, Broghammer N, Poets C. Fortschritte in der Neonatologie Vom Sicherstellen des Überlebens hin zu einer guten Lebensqualität. Frühförderung interdisziplinär 2011; 30: 128-136 
[4] Steinhardt A, Zöllner N. Elternberatung in der Neonatologie - Stationäre frühe Hilfen bei Risikogeburten. In: Reichert J, Rüdiger M, (Hrsg.). Psychologie in der Neonatologie. Psychologisch-sozialmedizinische Versorgung von Familien Frühgeborener. Göttingen: Hogrefe; 2013: 140-153

[5] Brisch KH, Buchheim A, Köhntop B et al. Präventives psychotherapeutisches Interventionsprogramm für Eltern nach der Geburt eines sehr kleinen Frühgeborenen - Ulmer Modell. Monatsschrift Kinderheilkunde 1996; 144: 1206-1212

[6] Mühler K, Pulzer F, Robel-Tillig E et al. Case-Management in der psychosozialen Begleitung von Eltern Frühgeborener und kranker Neugeborener. Kinder- und Jugendmedizin 2009; 9: 71-76

[7] Sommerfeld M, Fischer F, Freymann A. Elternschulung für Eltern frühgeborener Kinder im FamilieNetz. In: Reichert J, Rüdiger M, (Hrsg.). Psychologie in der Neonatologie. Psychologisch-sozialmedizinische Versorgung von Familien Frühgeborener. Göttingen: Hogrefe; 2013: 154-166

[8] Reichert J. Psychologisch-sozialmedizinische Versorgung im Stationären Bereich - Dresdner Modell. In Reichert J, Rüdiger M, (Hrsg.). Psychologie in der Neonatologie. Psychologisch-sozialmedizinische Versorgung von Familien Frühgeborener. Göttingen: Hogrefe; 2013: 238-254

[9] Panagl C, Kohlhauser C, Pollak A. Integrative psychologische Elternbetreuung auf der neonatologischen Intensivstation: Konzepte und erste Erfahrungen. Zeitschrift Geburtshilfe und Neonatologie 2005; 209: 14-21

[10] Scheithauer $H$, Petermann F. Frühinterventionen und -präventionen im Säuglings-, Kleinkind- und frühen Kindesalter. In: Petermann F, Niebank K, Scheithauer H, (Hrsg.). Risiken in der frühkindlichen Entwicklung. Entwicklungspsychopathologie der ersten Lebensjahre (S. 331-356). Göttingen: Hogrefe; 2000: 331-356

[11] Jotzo M, Reichert J. Psychoneonatologie - Ein Epilog. In: Reichert ], Rüdiger M, (Hrsg.). Psychologie in der Neonatologie. Psychologischsozialmedizinische Versorgung von Familien Frühgeborener. Göttingen: Hogrefe; 2013: 266-276

[12] Scheithauer H, Petermann F, Niebank K. Frühkindliche Entwicklung und Entwicklungsrisiken. In: Petermann F, Niebank K, Scheithauer H, (Hrsg.). Risiken in der frühkindlichen Entwicklung. Entwicklungspsychopathologie der ersten Lebensjahre. Göttingen: Hogrefe; 2000: $15-38$

[13] Scheithauer H, Niebank K, Petermann F. Biopsychosoziale Risiken in der frühkindlichen Entwicklung: Das Risiko- und Schutzfaktorenkonzept aus entwicklungspsychopathologischer Sicht. In: Petermann F, Niebank K, Scheithauer H, (Hrsg.). Risiken in der frühkindlichen Entwicklung. Entwicklungspsychopathologie der ersten Lebensjahre. Göttingen: Hogrefe; 2000: 65-97

[14] Bengel J, Lyssenko L. Resilienz und psychologische Schutzfaktoren im Erwachsenenalter. Stand der Forschung zu psychologischen Schutzfaktoren von Gesundheit im Erwachsenenalter. Forschung und Praxis der Gesundheitsförderung. Köln: Bundeszentrale für gesundheitliche Aufklärung; 2012 Band 43

[15] Rutter M. Resilience concepts and findings: implications for family therapy. Journal of family therapy. Blackwell publishers 1999; 21: 119-144

[16] Suess G, Zimmermann P. Anwendung der Bindungstheorie und Entwicklungspsychopathologie. Eine neue Sichtweise für Entwicklung und (Problem-) Abweichung. In: Suess GJ, Scheuerer-Englisch $\mathrm{H}$, (Hrsg.). Bindungstheorie und Familiendynamik. Giessen: Psycho-Sozial; 2001: 241-270

[17] Fröhlich-Gildhoff K, Rönnau-Böse M. Resilienz. (3. Auflage) München: Reinhardt; 2014

[18] Fehrenbach L. Frühgeborene in der Familie. Deutsche Hebammen Zeitschrift 2012; 4: 13-16
[19] Carlitscheck JC. Familienzentrierte Betreuung in der Neonatologie - Situationsanalyse und Zukunftsperspektiven. Inaugural-Dissertation, Fachbereich Humanwissenschaften, Köln. 2013; Zugriff am 08.02.15 unter http://unimedia.uni-koeln.de/2013/Humanwissenschaften/ Familienzentrierte_Betreuung.pdf

[20] Gloger-Tippelt G. Schwangerschaft und erste Geburt. Psychologische Veränderungen der Eltern. Stuttgart: Kohlhammer; 1988

[21] Christ-Steckhan C. Elternberatung in der Neonatologie. München: Reinhardt; 2005

[22] Hantsche B, Henze KH, Piechotta G. Psychosoziale Aspekte bei der Frühgeburt eines Kindes - eine Bestandsaufnahme. Praxis der Kinderpsychologie und Kinderpsychiatrie 1992; 41: 129-139

[23] Sarimski K. Frühgeburt als Herausforderung. Göttingen: Hogrefe; 2000

[24] Affleck G, Tennen H, Rowe J. Infants in crisis. How parents cope with newborn intensive care and its aftermath. New York: Springer; 1991

[25] Jotzo M, Schmitz B. Traumatisierung der Eltern durch die Frühgeburt ihres Kindes. Psychotraumatologie 2002; 3: 38

[26] Hynan MT, Mounts KO, Vanderbilt DR. Screening parents of high-risk infants or emotional distress: rationale and recommendations. Journal of Perinatology 2013; 33: 748-753

[27] Flatten G, Bär O, Becker K et al. S2 - Diagnostik und Behandlung von akuten Folgen psychischer Traumatisierung. Trauma und Gewalt 2011; 3: 214-221

[28] Hall S, Hynan M, Phillips R et al. Development of Program Standards for psychosocial Support of Parents of Infants Admitted to a Neonatal Intensive Care Unit; A National Interdisciplinary Consensus Model. Newborn \& Infant Nursing Reviews 2015; 15: 24-27

[29] Busse M, Stromgren K, Thorngate L et al. Parents' responses to stress in the neonatal intensive care unit. Critical Care Nurse 2013; 33: $52-60$

[30] Brisch KH. Prävention durch prä- und postnatale Psychotherapie. In: Brisch KH, Hellbrügge TH, (Hrsg.). Die Anfänge der Eltern-Kind-Bindung. Schwangerschaft, Geburt und Psychotherapie. Stuttgart: Klett-Cotta; 2007: 174-195

[31] Brisch KH, Buchheim A, Köhntop B et al. Präventives psychotherapeutisches Interventionsprogramm für Eltern nach der Geburt eines sehr kleinen Frühgeborenen - Ulmer Modell. Monatsschrift Kinderheilkunde 1996; 144: 1206-1212

[32] Cierpka M. Frühe Kindheit 0-3 Jahre. Beratung und Psychotherapie für Eltern mit Säuglingen und Kleinkindern. (2. Auflage) Berlin: Springer Medizin; 2014: 353-363

[33] Gebker S. Belastungsfolgen nach Frühgeburt: Die patho- und salutogene Wirkung des Scham- und Schulderlebens und der persönlichen Resilienz auf das mütterliche Wohlbefinden. Dissertation, Fachbereich Humanwissenschaften. Osnabrück. 2010; Zugriff am 08.02.15 unter http://repositorium.uni-osnabrueck.de/bitstream/urn: nbn:de:gbv:700-201011056688/7/thesis_gebker.pdf

[34] Scheithauer H, Petermann F. Zur Wirkungsweise von Risiko- und Schutzfaktoren in der Entwicklung von Kindern und Jugendlichen. Kindheit und Entwicklung 1999; 8: 13-14

[35] Mietzel G. Wege in die Entwicklungspsychologie. Weinheim: Beltz; 2002: $126-130$

[36] Schorn A. Bindungsorientierte Frühprävention und das Medium Video. 2007; Zugriff am 29.01.15 unter: http://www.kinderschutzbund-sh. de/tl_files/DKSB/Fachtage\%20und\%20Fortbildungen/Dokumentation/ Dokumentation \%20bis \%202008/06.11.07 + 06\%20-\%20Fruehe \%20 Hilfen, \%20Methoden/Bindungsorientierte\%20Fruehpraevention.pdf

[37] Ainsworth MDS. Muster von Bindungsverhalten, die vom Kind in der Interaktion mit seiner Mutter gezeigt werden (1964). In: Grossmann KE, Grossmann K. Bindung und menschliche Entwicklung. John Bowlby, Mary Ainsworth und die Grundlagen der Bindungstheorie. 3. AuflageStuttgart: Klett-Cotta; 2011: 102-111 
[38] Ainsworth MDS. Feinfühligkeit versus Unfeinfühligkeit gegenüber den Mitteilungen des Babys (1974). In: Grossmann KE, Grossmann K. Bindung und menschliche Entwicklung. John Bowlby, Mary Ainsworth und die Grundlagen der Bindungstheorie. 3. Aufl. Stuttgart: Klett-Cotta; 2011: 415-421

[39] Di Pietro JA. Psychological and psychophysiological considerations regarding the maternal-fetal relationship. Infant and child development 2010; 19: 27-38

[40] Brisch KH. Bindung und Trauma - Schutzfaktoren und Risiken für die Entwicklung von Kindern. Psychotherapie im Dialog 2006; 4: 382-386

[41] Rüdiger M. Frühgeburt: Von der medizinischen Behandlung des Kindes zur Begleitung seiner Familie. In Reichert J, Rüdiger M, (Hrsg.). Psychologie in der Neonatologie. Psychologisch-sozialmedizinische Versorgung von Familien Frühgeborener. Göttingen: Hogrefe; 2013: 29-38

[42] Mehler K, Wendrich D, Kißgen R et al. Mothers seeing their VLBW infants within $3 \mathrm{~h}$ after birth are more likely to establish a secure attachment behavior: evidence of a sensitive period with preterm infants? Journal of Perinatology 2011; 31: 404-410

[43] Wigert H, Johansson R, Berg MD et al. Mothers ' experience of having their newborn child in a neonatal intensive care unit. Scandinavian Journal of Caring Sciences 2006; 20: 35-41

[44] Bundesverband „Das frühgeborene Kind“ e. V.. Elternberatung in der Neonatologie. Frankfurt am Main: Volkhardt Caruna Medien GmbH; 2014 Zugriff am 12.02.15 unter http://www.fruehgeborene.de/sites/ default/files/field_page_file/leitfaden_elternberatung_a_5_28._april_2014_web.pdf

[45] Nestmann F. Beratung. Bausteine für eine interdisziplinäre Wissenschaft und Praxis. Tübingen: DGTV; 1997: 33-35

[46] Kißgen R, Carlitscheck J, Rapp C et al. Die psychosoziale Versorgung in der Neonatologie in Deutschland: Eine quantitativ-empirische Bestandsaufnahme aus ärztlicher Perspektive. Zeitschrift für Geburtshilfe und Neonatologie 2012; 216: 259-268

[47] Reichert J, Rüdiger M. Psychologie in der Neonatologie. Psychologischsozialmedizinische Versorgung von Familien Frühgeborener. Göttingen: Hogrefe; 2013: 7-8

[48] Machul D. Frühgeburt: Elterlicher Bedarf an professioneller Unterstützung während des Klinikaufenthaltes. Dissertation, Fachbereich Rehabilitationswissenschaften, Berlin. 2008; Zugriff am 08.02.14 unter http://edoc.hu-berlin.de/dissertationen/machul-doerthe-2008-10-27/ PDF/machul.pdf

[49] Reuschenbach B, Mahler C. Pflegebezogene Assessmentinstrumente: Internationales Handbuch für Pflegeforschung und -praxis. Bern: Hans Huber; 2011: 27-29
[50] Meyer EC, Lester BM, Boukydis CFZ et al. Family-based intervention with high-risk infants and their families. Journal of Clinical Psychology in Medical Settings 1998; 5: 49-69

[51] Miles M, Funk S, Carlson J. Parental stressor scale: Neonatal care unit. Nursing research 1993; 10: 243-253

[52] Meyer EC, Zeanah CH, Boukydis FZ et al. A clinical interview for parents of high-risk infants: Concept and applications. Infant Mental Health Journal 1993; 14: 192-207

[53] Mok E, Leung SF. Nurses as providers of support for mothers of premature infants. Journal of clinical nursing 2006; 15: 726-734

[54] Aarnoudse-Moens CSH, Weisglas-Kuperus N, van Goudoever JB et al. Meta-Analysis of neurobehavioral outcomes in very preterm and/or very low birth weight children. Pediatrics 2009; 124: 717-728

[55] Baron IS, Rey-Casserly C. Extremely preterm birth outcome: a review of four decades of cognitive research. Neuropsychology Review 2010; 20: $430-452$

[56] Bengel J, Meinders-Lücking F, Rottmann N. Schutzfaktoren bei Kindern und Jugendlichen - Stand der Forschung zu psychosozialen Schutzfaktoren für Gesundheit. Forschung und Praxis der Gesundheitsförderung, Band 35. Köln: Bundeszentrale für gesundheitliche Aufklärung; 2009

[57] Laucht M, Schmidt MH. Entwicklungsverläufe von Hochrisikokindern: Ergebnisse der Mannheimer Längsschnittstudie. Kinderärztliche Praxis 2005; 76: 348-354

[58] Meyer-Probst B, Reis O. Risikofaktoren und Risikobewältigung im Kontext: Schlußfolgerungen aus der Rostocker Längsschnittstudie nach 25 Jahren. Frühförderung interdisziplinär 2000; 19: 109-118

[59] Sarimski K, Hintermair M, Lang M. Familienorientiertes Arbeiten in der Frühförderung. Perspektiven von Fachkräften. Frühförderung interdisziplinär 2014; 33: 68-79

[60] Jotzo M. Trauma Früh- und Risikogeburt. In: Reichert J, Rüdiger M, (Hrsg.). Psychologie in der Neonatologie. Psychologisch-sozialmedizinische Versorgung von Familien Frühgeborener. Göttingen: Hogrefe; 2013: 112-139

[61] Saunders RP, Abraham MR, Crosby M] et al. Evaluation and development of potentially better practices for improving family-centred care in neonatal intensive care units. Pediatrics 2003; 111: 437-449

[62] Hüning BM, Reimann M, Beerenberg $U$ et al. Establishment of a family-centred care programme with follow-up home visits: Implications for clinical care and economic characteristics. Klinische Pädiatrie 2013; 224: 431-436

[63] Vonderlin EM, Nöcker-Ribaupierre M, Wilke M. Unterstützende Behandlungsangebote in der Neonatologie. Neonatologische Abteilungen in Deutschland. Monatsschrift Kinderheilkunde 2009; 158: $42-47$ 